\title{
Interactive Hairstyle Modeling Using a Sketching Interface
}

\author{
Xiaoyang $\mathrm{Mao}^{1}$, Kouichi Kashio ${ }^{2}$, Hiroyuki Kato ${ }^{1}$ and Atsumi Imamiya ${ }^{1}$ \\ ${ }^{1}$ Department of Computer and Media Engineering, Yamanashi University, 4-3-11 Takeda, \\ Kofu, Yamanashi 400-0016, Japan \\ $\{$ Mao, kato, imamiya $\}$ Qhci.media.yamanashi.ac.jp
Sony Conporation, Mobile Netw ork Com pany, Japan. \\ Hirokazu.Kashioddvpj.sony.co.jp
}

\begin{abstract}
Modeling and rendering human hair remains to be one of the most challenging computer graphics problems. This paper presents a new interactive hair modeling system featured with a user-friendly sketching interface. With the sketching interface, any user, even a first time user, can create a hair model of his/her desired style just in a few minutes simply by interactively drawing several strokes illustrating the global and local features of the hairstyle. The result of the system is a generic geometry-based representation of hair which can be post manipulated and rendered with most existing systems.
\end{abstract}

\section{Introduction}

Needless to say, hair is one of the most important visual elements featuring a human character. A person can look having a completely different expression just by changing his/her hairstyle. To synthesis realistic hair images, researchers have been challenged by many difficult problems inherently caused by the special properties of human hair, such as the huge number of hair strands, the extremely small diameter of individual strand compared with an image pixel, and the complex interaction of light and shadow among strands. In the past decade, a number of novel techniques have been developed for successfully shading hair strands and eliminating alising artifacts[1 6]. In this paper, we presents a new system for allowing users to interactively create 3D hair models of their desired styles. Modeling different 3D hairstyles is even a more difficult problem as the huge variation of hairstyles is determined by extremely complex interactions of many factors. These factors include gravity, static charges, strand-to-strand and strand-to-head interaction, styling tools such as hair pins, hair bands and mousse. Recently, several papers have been published addressing this hairstyle modeling issue[7 11]. A common feature of those systems is the using of cluster model. Because of the cosmetics and static electricity, nearby hair strands tend to form clusters. Use a cluster instead of a single strand as the smallest unit of shape control makes it possible for allowing users to interactively design their desired hairstyles. The trigonal prism wisp model presented by Chen et al.[7] represents each cluster(wisp) with a trigonal prism and the hair strands within a cluster is modeled by the distribution map defined on the cross-sections of the prism. 
An editing tool is provided for allowing users to interactively position clusters on the scalp and change the shapes of clusters. The hierarchical cluster model and VhairStudio developed by $\mathrm{Xu}$ et al.[8,10] uses a generalized cylinder to represent a hair cluster. Each cross-section of a cylinder is associated with a density distribution of the hair strands inside the cluster and the output of the system is a volume density representation of hair. Hairstyles are created by interactively changing the shape of the center lines of cylinders. Other researchers explored the possibility of using vector fields for hairstyle modeling[9,11]. Yu succeeded in creating very realistic and complicated hairstyles by modeling the flow and curliness of hair using procedurally defined vector fields[11].

Although most existing hair modeling systems do provide users with some kinds of interactive design tools, the interfaces of those tools, however, are not user friendly. It can be very difficult and tedious for users to construct a hairstyle by adjusting the shape of each hair cluster. For example, it takes about one to two hours for a user familiar with the system to create a typical hairstyle with the hierarchical cluster model[8]. The largest advantage of our new hairstyle modeling system is its userfriendly sketching interface. With this interface, any user, even a first time user, can create a hair model of his/her desired style just in a few minutes. Our technique is based on the consideration that when we look at someone's hairstyle, we pay more attention to its overall design instead of the detailed shape of individual hair strand or cluster. Therefore, instead of asking a user to put strands or clusters together to build up the whole hairstyle, we ask a user to start with specifying the silhouette of the target hairstyle. Based on the silhouette, the system automatically generates a hairstyle and then the user is allowed to locally modify it to obtain the ideal one. In our system, the hairstyle silhouette and all other operations are specified interactively with 2D mouse or tablet as freeform strokes.

The remaining part of the paper is organized as follows: Section 2 gives the overview of the system. Section 3 describes how to specify the silhouette and partition lines of hair and also the area for growing hair. Section 4 describes the algorithm for defining the surface boundary of a hairstyle and Section 5 introduces the rule for growing hair. Section 6 demonstrates some hairstyles generated with our system. Finally, in Section 7, we conclude the paper by showing the future research directions.

\section{Overview}

A typical hairstyle modeling process in our system consists of the following 6 steps:

1. Loading 3D head model to the view window

2. Specifying the area to grow hair

3. Specifying the partition line

4. Specifying the silhouette of the hairstyle

5. Growing hair

6. Locally modifying the hairstyle

After the 3D head model is displayed on the view window, a user is asked to interactively draw the boundary of the region to grow hair and also the partition line 
of hair. Fig. 1(a) is an example of such a user-drawn boundary and a partition line. Next, the user is asked to draw a stroke representing the silhouette of the target hairstyle(Fig. 1(b)). Based on the silhouette line, the system automatically generates a surface representing the boundary of the hairstyle(Fig. 2(b)). We call this surface silhouette surface hereafter. $3 \mathrm{D}$ hair model is then obtained by growing hair strands to fill in the space between the silhouette surface and the surface pf the scalp(Fig. 1(d)). Finally, the user is allowed to locally modify the hairstyle using operations such as cutting, combing and perm. Currently, only the cutting operation is supported and other local modification operations will be available in the near future. We will discuss the detail of each step in the following sections.

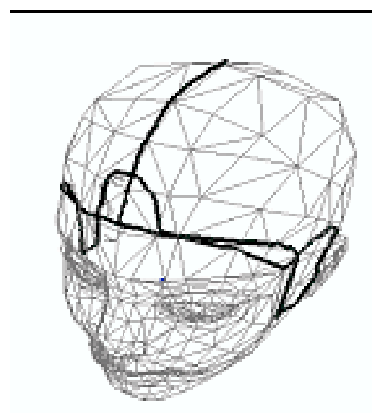

$(3)$

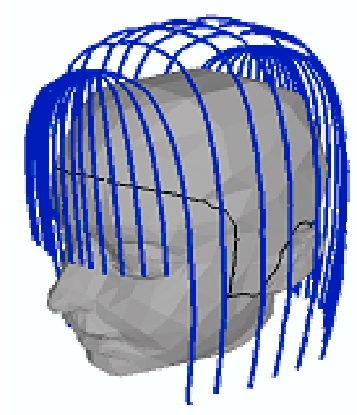

(c)

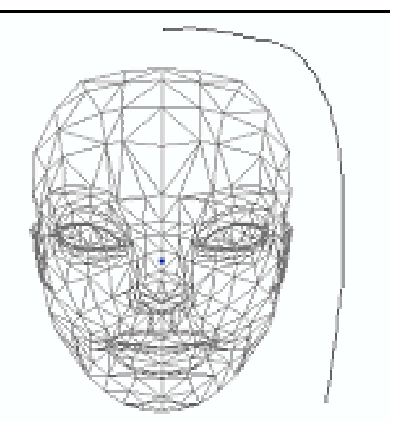

(b)

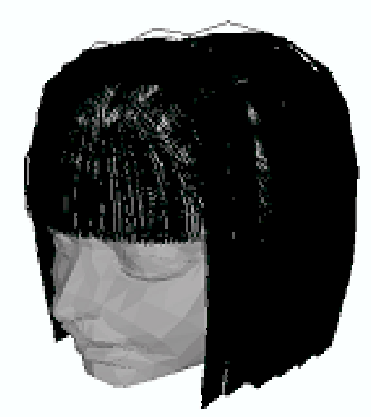

di

Fig. 1. A typical process of hairstyle modeling. (a) A user draws the strokes specifying the boundary of the region to grow hair and the partition line of hair. (b) The user also draw the silhouette line of the target hairstyle. (c) The system automatically creates the silhouette surface of the hairstyle. (d) The system grows hair strands to fill in the space between the silhouette surface and the surface of the scalp. 


\section{Specifying the Region to Grow Hair, the Partition Line and the Silhouette Line}

The boundary of the region to grow hair, the partition line of hair and the silhouette of a hairstyle are all specified through drawing freeform strokes either using a 2D mouse or a tablet. The strokes representing the boundary of the region to grow hair and the partition line are drawn on the displayed 3D head model directly. A user can rotate the head model to any angle suitable for the drawing task. As shown in Fig. 2, the drawn strokes are projected onto the head model to get the 3D representation of the boundary and the partition line.

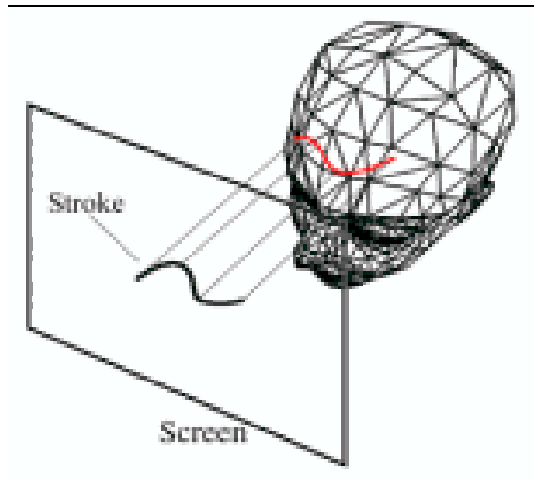

Fig. 2. Strokes drawn on the view window are projected onto the 3D head model.

To find the region inside the boundary, we need a flattened map of the scalp. A possible approach is to parameterize the scalp using a $2 \mathrm{D}$ polar coordinate system[11]. Here we use projection to map the boundary and the scalps onto a 2D plane. We virtually place a plane on the top of the head and project the boundary as well as all the vertices of the head model onto the plane. By carefully selecting the position of the projection center, we can preserve the relative 3D position of vertices respective to the boundary on the $2 \mathrm{D}$ plane. Currently, our system only accepts partition line located at the center of the top of the scalp and we assume the resulting hairstyle is symmetric about the partition line. Therefore users are asked to draw only the right half silhouette of a hairstyle.

\section{Silhouette Surface}

Based on the silhouette line, the system automatically creates the silhouette surface of the hairstyle. The shape of the silhouette surface should be decided by taking into consideration both the shape of silhouette line and the shape of scalp. To do this, as shown in Fig. 3, we first obtain a set of evenly spaced cross-sections of the head model along the Z-axis and create a silhouette line for each cross-section by deforming the input silhouette line to match the shape of the contour line of the cross- 
section. The cross-section starts at the front end point of the partition line and ends at the back end point. Interpolating the silhouette lines for all cross-sections gives the silhouette surface of the side area of the hairstyle. The cross-section with the largest width in X-direction is called basic cross-section. The user drawn silhouette line is first projected onto the plane of the basic cross-section to obtain the silhouette line for the basic cross-section. We call this silhouette line basic silhouette line. To create the silhouette line for other cross-sections, we calculate the height and width difference of the cross-section and the basic cross-section. Then the silhouette line is obtained by first translating the basic silhouette line in Y-direction the amount of the height difference followed by a shrinking in X-direction with the amount of the width difference.

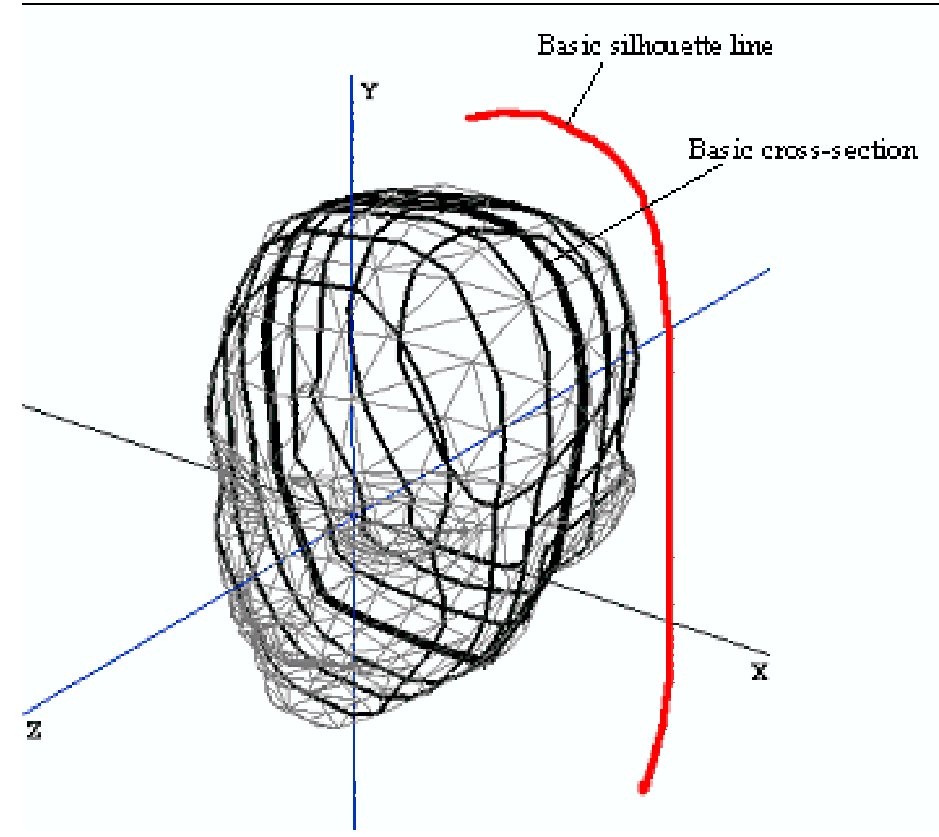

Fig. 3. Cross-sections and silhouette lines.

To generate the silhouette surface for the forehead and the back area, we approximately assume hair strands grow radially from the front and back end points of the partition line. As shown in Fig. 4, to obtain the silhouette lines for the front area, we sweep the silhouette line for the cross-section at the front end point of the partition line through the forehead while keeping the distance from the silhouette lines to the forehead to be constant. The silhouette lines for the back area is created in the same way by sweeping the silhouette line for the cross-section at the back end point of partition line. Fig. 1(c) is the silhouette surface generated with the described method based on the user drawn silhouette line shown in Fig. 1(b). 


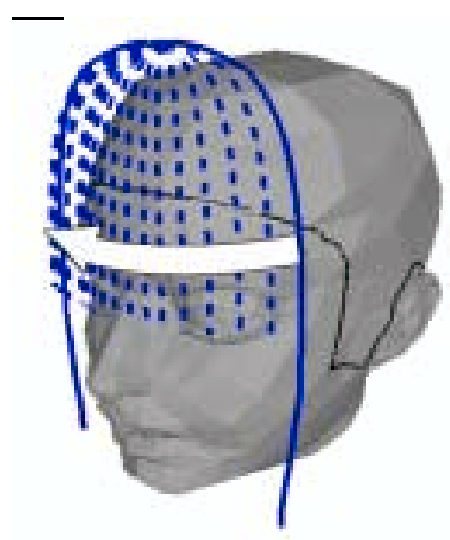

Fig. 4. Creating silhouette lines for the forehead area.

\section{Growing Hair}

After the surface silhouette of a hairstyle is generated, the system automatically grows hair to fill in the space between the silhouette surface and the surface of the scalp. There are mainly two existing approaches to represent hair. One is called explicit model and the other is called volume density model. The explicit model represents individual hair strand with some geometrical object, such as a connection of cylinders, a curved polyline or a connected segments of triangular prism[1,2,4,5,11] while the volume density model represents the entire hair style macroscopically as a 3D distribution function of hair density[3,8,10]. Our system chooses the explicit model and use curved polyline to represent each hair strand. We choose the explicit model because most existing hair modeling and rendering systems support this model rather than the volume density model, and therefore the resulting hair models of our system can be easily post manipulated and rendered with those systems. Usually at least 10000 to 15000 hair strands are required to generate a realistic hair image. On the other hand, the number of hair strands used in the modeling stage should be as small as possible so as to provide real time feedbacks to user's operations. To fulfill these two requirements simultaneously, we allow users to specify the number of hair strands by themselves. As we will demonstrate in the next section, 1000 strands seems to be enough for the purpose of previewing and with this number users can get a real time feedback of their operations on a standard PC. We also support a trigonal prism representation for the final hair model.

After the number of hair strands is specified, the positions for growing these hair strands are decided by uniformly distributing this number of points over the scalp within the region to grow hair. Each hair strand is generated following the rule that it passes through the space in between the silhouette surface and the surface of the scalp. The higher its growing position is, the closer it is from the silhouette surface. This rule is depicted in Fig. 5. We first generate the vertical line segment between the top of the scalp and the lowest position of hair growing region. Then we bisect the 
segment with the position to grow the strands to obtain two segments of length a and $\mathrm{b}$, respectively. The polyline of the hair strand is then constituted by the vertices bisecting the distance between the silhouette surface and scalp surface by the ration of $\mathrm{a} / \mathrm{b}$.

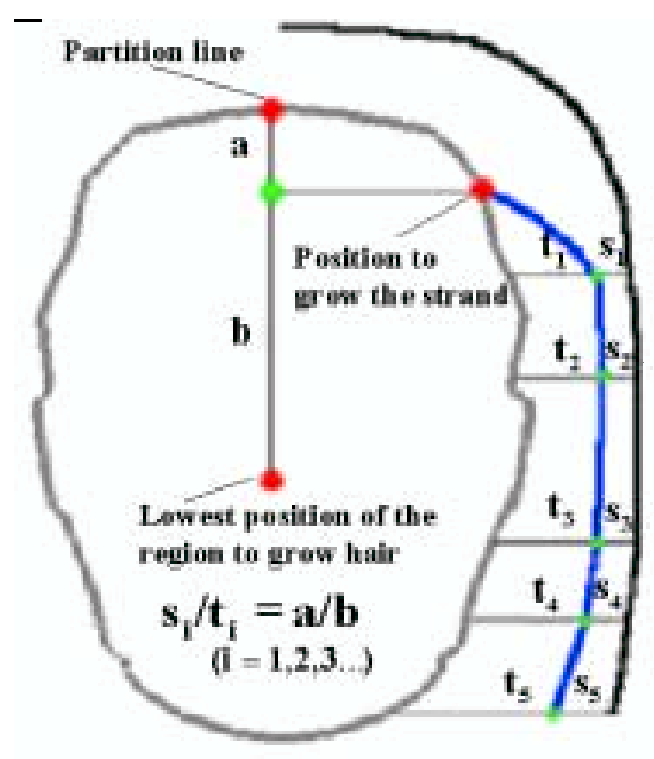

Fig. 5. Rule for growing a hair strand.

\section{Results}

We have implemented our interactive hairstyle modeling system on Windows environment with Visual C++ and OpenGL graphics library. Fig. 6 shows several images of the hairstyle generated with the stroke inputs shown in Fig. 1. Fig. 6(a) and (b) are the images when 1000 and 10000 hair strands are used, respectively. Fig. 6(c) is the image generated by locally applying a cutting operation to the hair model shown in Fig. 6(b). Cutting operation is specified by drawing the freeform stroke of the cutting line directly on the 3D rendered hair model. The Fig. 6(d) is the image rendered with 3D StudioMax ${ }^{1}$. Each hair strand is represented as a trigonal prism in this case. Times required for rendering images of 1000 and 10000 hair strands on a Pentium machine with two Xeon $933 \mathrm{MHz}$ CPU are about 0.4 and 9 seconds, respectively. Fig. 7 and 8 demonstrate that how curly and wavy hairstyle can also be easily created simply by drawing a curly or wavy silhouette line.

1 3D StudioMax is the trade mark of Autodesk, Inc. 


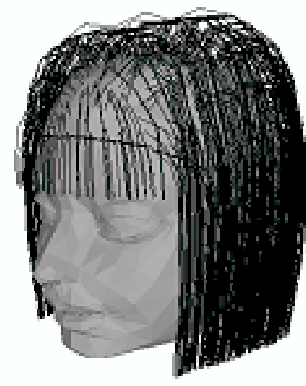

(a) 1000 strands

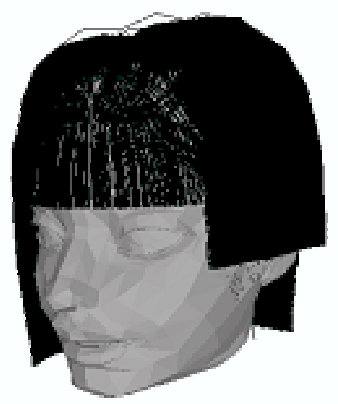

(c) local cutting

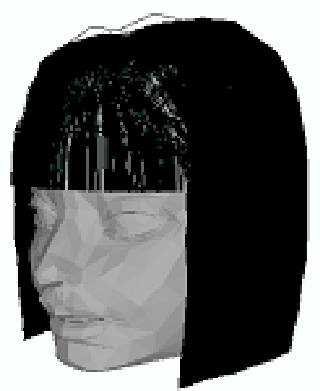

(b) 10000

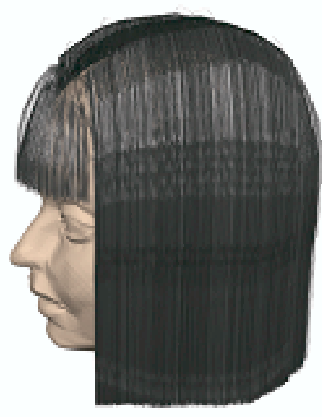

(d) shaded image

Fig. 6. Images generated with the stroke inputs shown in Fig. 1.
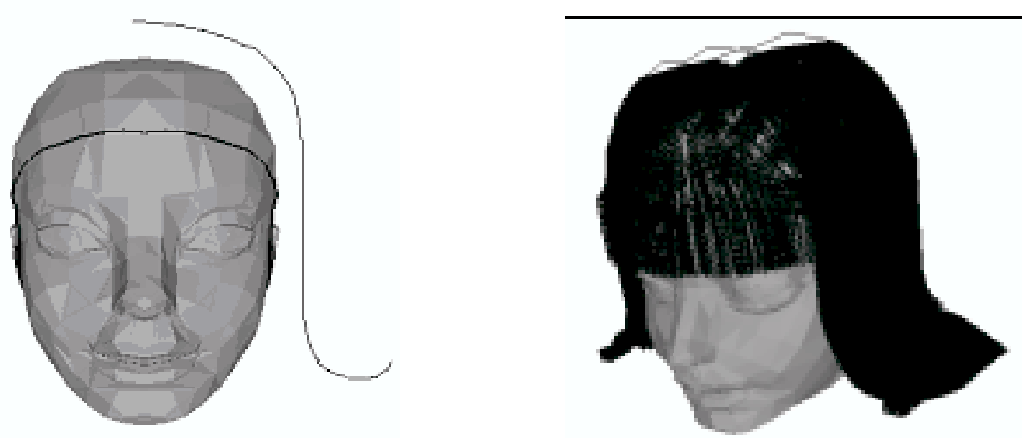

Fig. 7.A curly hairstyle generated with our system. Left: user specified silhouette. Right: resulting hairstyle. 

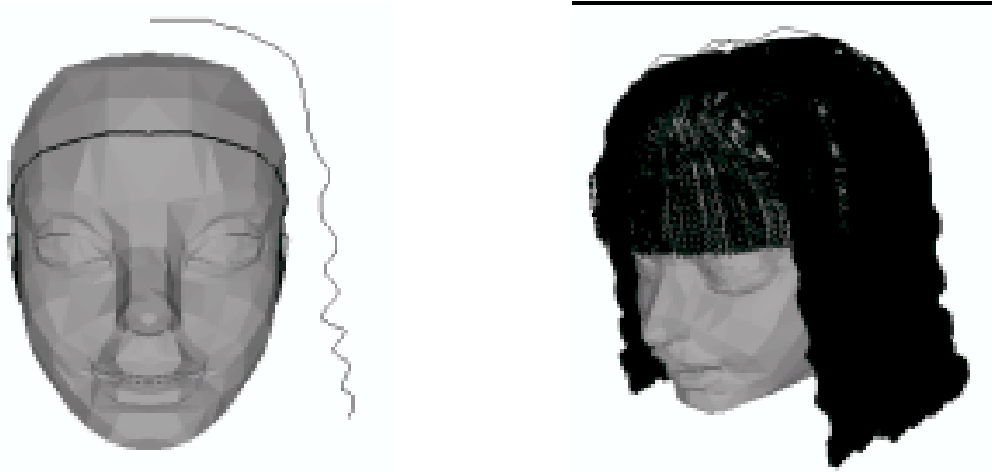

Fig.8. A wavy hairstyle generated with our system. Left: user specified silhouette. Right: resulting hairstyle.

\section{Concluding Remarks}

We have presented an interactive hairstyle modeling system featured with a sketch interface. The idea of using sketching interface is also inspired by Teddy, a successful sketching interface for 3D freeform design[12]. As we mentioned in Section 1, the variation of hairstyles is determined by extremely complex interactions of many factors, such as gravity, strand-to-strand and strand-to-head interaction and styling tools. A faithful physical simulation of all those factors can be very difficult and time consuming. In many applications, however, an expressive presentation of personalized features of hairstyles is of most important. Our sketching interface provides an easy to use expressive design tool to those applications. For example, as the potential applications, our system can be used to smooth the communication between a customer and a stylist at hair salons and also used for in house CG creation for visually present the a designer's requirements to a professional CG modeling specialist.

The results presented in this paper are just the very initial results of our prototype system and the variation of hairstyles is limited currently. We are now implementing other local modification operations such as combing and perm. To make the hair image look more realistic, we need to add some randomness to the positions and shapes of hair strands generated following the rule introduced in Section 5. We are now implementing a method which groups hair strands near by into clusters and adds randomness to the shape of each hair cluster. Also we need some special consideration for generating short cut hairstyles. 


\section{Acknowledgements}

The authors would like to thank Issei Fujishiro from Ochanomizu University for his helpful comments and continuous support. This work was partially supported by Telecommunications Advancement Organization of Japan.

\section{References}

1. LeBlanc A. M., Turner R. and Thalmann D.: Rendering Hair using Pixel Blending and Shadow Buffers, The Journal of Visualization and Computer Animation, Vol. 2, (1991) 9297

2. Rosenblum R.. R., Carlson W. E. and Tripp III E.: Simulating the Structure and Dynamics of Human Hair: Modeling, Rendering and Animation, The Journal of Visualization and Computer Animation, Vol. 2, (1991)141-148

3. Kajiya T. and Kay T.L.: Rendering Fur with Three Dimensional Textures, Computer Graphics, Vol.23, No.3, (1989) 271-280

4. Watanabe Y. and Suenaga Y.: A Trigonal Prism-Based Method for Hair Image Generation", IEEE Computer Graphics \& Applications, (1992) 47-53

5. Anjyo K., Usami Y. and Kurihara T.:A Simple Method for Extracting the Natural Beauty of Hair, Computer Graphics, Vol.26, No.2, (1992) 111-120

6. Magnenat Thalmann N., Kurihara T., Thalmann D.: An Integrated System for Modeling, Animating, and Rendering Hair., Proceedings of EUROGRAPHICS '93, 211-221

7. Chen L.H., Saeyor S., Dohi H. and Ishizuka M.: A System of 3D Hair Style Synthesis based on the Wisp Model, Visual Computer, Vol. 15, (1999) 159-170

8. Yang, Xue Dong, Tao Wang Zhan $\mathrm{Xu}$, and Hu Qiang: Hierarchical Cluster Hair Model, Proceedings of the IASTED International Conference on Computer Graphics and Imaging, (2000) 75-81

9. Hadap S. and Magnenat-Thalmann N.: Interactive Hair Styler Based on Fluid Flow, Proceedings of Eurographics Workshop on Computer Animation and Simulation '2000

10.Xu Z. and Yang X. D: V-HairStudio: An Interactive Tool for Hair Design, IEEE Computer Graphics \& Applications, Vol. 21, No. 3 (2001)36-43

11.Yu Yizhou: Modeling Realistic Virtual Hairstyles,Proceedings of Pacific Graphics'01, (2001) 295-304

12.Igarashi T., Matsuoka S., Tanaka H.: Teddy: A Sketching Interface for 3D Freefrom Design, ACM SIGGRAPH 99, 409-416 